\title{
Investigations of reconstruction of passivation layer on stainless steel surface using AFM-based techniques
}

\author{
Marlena BŁaszczyK ${ }^{1}$, Martyna Durko ${ }^{1, *}$, ZuZAnna IWANickA $^{1}$, PAWEŁ Lochyński ${ }^{2}$, \\ ANDRZEJ SIKORA ${ }^{3}$ \\ ${ }^{1}$ Active Students Association, SEP Branch No. 1 in Wrocław, M. Skłodowskiej-Curie 55/61, 50-369 Wrocław, Poland \\ ${ }^{2}$ Institute of Environmental Engineering, Wrocław University of Environmental and Life Sciences, \\ Grunwaldzka 55, 50-357 Wrocław, Poland \\ ${ }^{3}$ Division of Electrotechnology and Materials Science, Electrotechnical Institute, \\ M. Skłodowskiej-Curie 55/61, 50-369 Wrocław, Poland
}

\begin{abstract}
Continuous development of stainless steel technology forced by the increase in the growing demands on the operating parameters of various stainless steel alloys, is the motivation for implementation of research for understanding the complexity of electrochemical processes ongoing on the surface of a material during various technological processes and during exploitation of the finished components. In this paper, the use of atomic force microscopy (AFM) is presented as a tool for observation of reconstruction process of passivation layers on the surface of previously electropolished stainless steel. For this purpose, a technique called nanoscratching was used, in which scratches are made on the surface of a material by means of diamond scanning probe, which violates the continuity of the passivation layer. Then, the dynamics of the process of reconstruction of that layer was assessed using continuous imaging of the scratched area in AFM semicontact mode. Studies of this type can be used to evaluate the impact of various factors on the spontaneous reconstruction of the passivation layer as well as possible susceptibility of the material on the course of corrosion processes initiated as a result of mechanical defects arising during operation of the material. By using the AFM, it was possible to observe changes in the depth of scratches with a subnanometer resolution. Obtained results proved that the presented AFM application allowed observation of the dynamics of passivation layer reconstruction process in a quantitative fashion, therefore it seems to be a very useful tool in the investigation of the impact of various conditions on this phenomenon. The results showed that changes in surface modification were occurring in a continuous manner. Changing dynamics of said process was presented. It should be underlined that no such experiments have been reported so far.
\end{abstract}

Keywords: atomic force microscopy; passivation layer; stainless steel; nanoscratching

\section{Introduction}

In this paper, employing advanced AFM technique for observation of passivation layer reconstruction is presented. In the research, samples of stainless steel, previously subjected to electropolishing process, were used. Electropolishing is an electrochemical method of steel processing that involves removal of a metal layer from the surface of material which is immersed in the electrolyte solution. This layer is removed as a result of current flow through the electrical circuit. Purified material is connected as an anode in a galvanic bath being a part of this circuit. As a result, the exposed metal

*E-mail: martyna.durko@gmail.com layer is free of impurities. The main purpose of this technological process is purification and reduction of roughness of the material surface. However, due to anode passivation which occurs at the same time, the material is further protected against corrosion. The use of electrochemical polishing eliminates the stresses caused by mechanical polishing. As a result of electropolishing, a smooth, clean and shiny surface with improved chemical resistance is obtained [1-3]. The electropolished samples have been subjected to further testing. In our case, it was the observation of dynamics of reconstruction of the protective layer, whose continuity was destroyed by modifying the said surface in the process of nanoscratching. For this purpose, an atomic force microscope was used. 
AFM was invented in the eighties of the last century and it provides many possibilities to study and analyze the structure of a material, e.g. it is possible to scan a sample surface or to modify it and to obtain the results in a nanometer (or even subnanometer) scale. AFM can be used for quantitative evaluation of morphological changes of surface caused by the action of various factors, such as solar radiation, UV light, electric field, etc. By quantitative surface imaging in three dimensions, it is possible to determine the roughness parameters and to assess the dynamics of degradation processes [4-10]. With AFM it is possible to study the aging of material as well as to track the rebuilding process of outer layer after subjecting it to nanoscratching.

So far, a number of nanoscratchning applications was reported, e.g. nanolithography [11], investigation of scratch resistance [12], in process of manufacturing of ferromagnetic nanostructures [13], or as a means of polymer thickness measurement [14]. This method is also used to improve the quality of AFM imaging. Many measurements should be carried out to minimize errors arising from the presence of artifacts. However, to make results reliable and feasible for comparison, it is necessary to conduct tests always at exactly the same spot on the sample surface. For this purpose, nanomarkers are used, which are the marks made at selected locations on the surface of tested material. They allow unambiguous determination of the scanning area. For the measurements carried out using AFM, nanomarkers are made at the spots previously designated in the nanoscratching process, resulting in permanent deformation by the local pressure of the microscope probe on the surface. Nanomarkers application significantly reduces the dispersion of the studied parameters and also helps to improve the measurements accuracy $[15,16]$.

\section{Experimental}

Nanoscratching is a process in which nanoscratches are made on the surface of a sample, thereby, disrupting its consistency. To be able to perform cuts on the outer layer of material (in this work stainless steel), it is necessary to use a scanning probe with strength parameters and wear resistance good enough to allow scratching without damaging the probe. The advantage of the diamond or diamond coated tip makes it superior to standard silicon probes in this unique application despite significant price difference [17-19]. Nanoscratching should be done with particular caution: wrong choice of parameters can cause unexpected damage to the tested surface and the scanning probe. It makes the process time-consuming. Despite these inconveniences, this method is one of the best to studying the reconstruction processes. The thickness of the passivation layer is at the nanometer level, the scale in which other diagnostic techniques are ineffective. Moreover, by performing these measurements using AFM it is possible to observe how the change in environmental conditions (especially humidity) affects the modified layer [20]. A unique approach aimed at the investigation of the submicron features on the surface was reported, where a AFM probe was used in order to cause the wear and to measure its effect [21].

The research was carried out on samples of stainless steel, i.e. AISi 304. The chemical material composition in wt.\% was: $0.037 \mathrm{C}, 0.42 \mathrm{Si}, 0.057$ $\mathrm{N}, 1.28 \mathrm{Mn}, 0.029 \mathrm{P}, 0.002 \mathrm{~S}, 18.13 \mathrm{Cr}, 8.04 \mathrm{Ni}$ and balance Fe. The samples before the test were electropolished. The research was carried out using dynamic mode with the atomic force microscope Innova from Bruker using a diamond scanning probe. The investigation was performed at a constant room temperature approx. $25^{\circ} \mathrm{C}$ and humidity approximately $35 \%$. To make scratches on the material, NanoPlot program was used [22]. The analysis of the measurement data was carried out using the SPIP program [23].

Once a suitable place was selected arbitrarily, the scratches were made on the surface of the tested material with a force of a value approximately $8.4 \mu \mathrm{N}$ with the NanoPlot program. It has to be underlined, that due to mechanical inhomogeneity of the material, the force had to be adjusted experimentally, while the objective was to fabricate desired surface modification for further observations. In order to perform the work, only areas 
with a rather homogeneous surface were chosen. The next step was to perform continuous imaging of the said area for 24 hours, which made it possible to measure the depth changes of obtained modifications. The tests were carried out at a specific location of the scratch. The analysis of the measurement data was executed with the SPIP program.

Observation of the process of the passivation layer reconstruction was carried out using TappingMode with the same scanning probe that was used in the process of nanoscratching. Using the AFM, it was possible to observe changes in the depth of scratches on the subnanometer level. We used a DDESP probe from Bruker with following specific parameters (supplied by the producer): material: $0.01 \Omega \cdot \mathrm{cm}$ to $0.02 \Omega \cdot \mathrm{cm}$ antimony (n) doped silicon, back coating: aluminum, tip coating: conductive diamond, $\mathrm{r}_{\text {tip }}=150 \mathrm{~nm}, \mathrm{k}=42 \mathrm{~N} \cdot \mathrm{m}^{-1}$, $\mathrm{f}_{\text {res }}=320 \mathrm{kHz}$. Speed of the probe was set to $0.5 \mu \mathrm{m} / \mathrm{s}$ and the determined change of the probe height was set to $200 \mathrm{~nm}$. The actual force used to modify the surface varied according to the observed area due to different grain orientations of the passivation layer.

In order to improve the time-related observation of the scratches changes, the slow scanning axis was disabled. Such an approach enabled acquisition of a series of profiles in one place of the scratch, therefore the process dynamics could be observed with a time constant equal to single line acquisition. Therefore, the major AFM drawback, which is relatively slow surface scanning, could be overcome.

\section{Results and discussion}

According to the methodology described in the preceding paragraph, the study was carried out in several arbitrarily chosen areas of a stainless steel sample where an important criterion of surface selection was its smoothness. The example of acquired topography image of the sample is presented in Fig. 1. It is clearly visible that the depth of the scratches is not the same at every point of modification (the deepest point seem to be near the starting point, where the surface was scratched). The tests were performed at the point situated slightly lower than the halfway of the sample. Using SPIP program, the depth of the scratches was measured. The averaged profile was created using the data made of following 20 lines. It assured noise immunity of the readout. In Fig. 2, triangular markers, marking the place where the depth of the studied sample was measured are presented. Markers No. 2, No. 3, No. 6 and No. 7 relate to the depth of modifications, while the remaining ones (i.e. No. 1, No. 4 , No. 5 , No. 8) have been used to find the plateau. The data relating to the depth of each figure are shown in Fig. 3. Uncertainty of measurement does not exceed $5 \%$. Ridges visible in Fig. 2 and Fig. 3 mainly result from performed scratching and represent the parts of material surface that were taken off during the process. They are also visible due to passivation layer reconstruction.

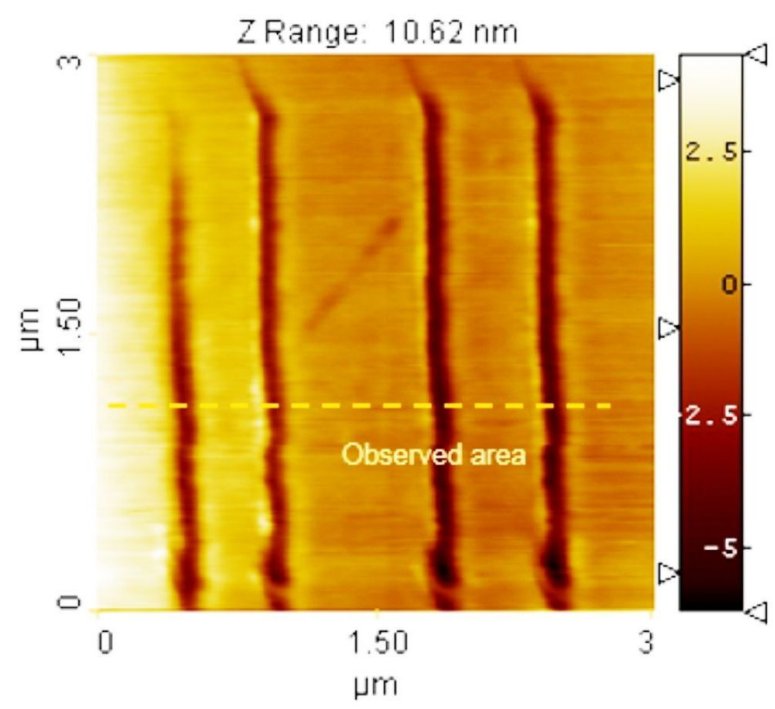

Fig. 1. AFM image of the investigated sample topography.

Fig. 4 and Fig. 5 show AFM images of surface modifications resulting from nanoscratching. These images present the changes in structure over the course of $1 \mathrm{~min}, 15 \mathrm{~min}$ and $24 \mathrm{~h}$ starting from the modification of the passivation layer. A small range of changes visible relatively easily is caused by the migration of the particles making up the said layer. The scratch depth measurement is possible through AFM imaging, while the obtained values come from the difference in height between 


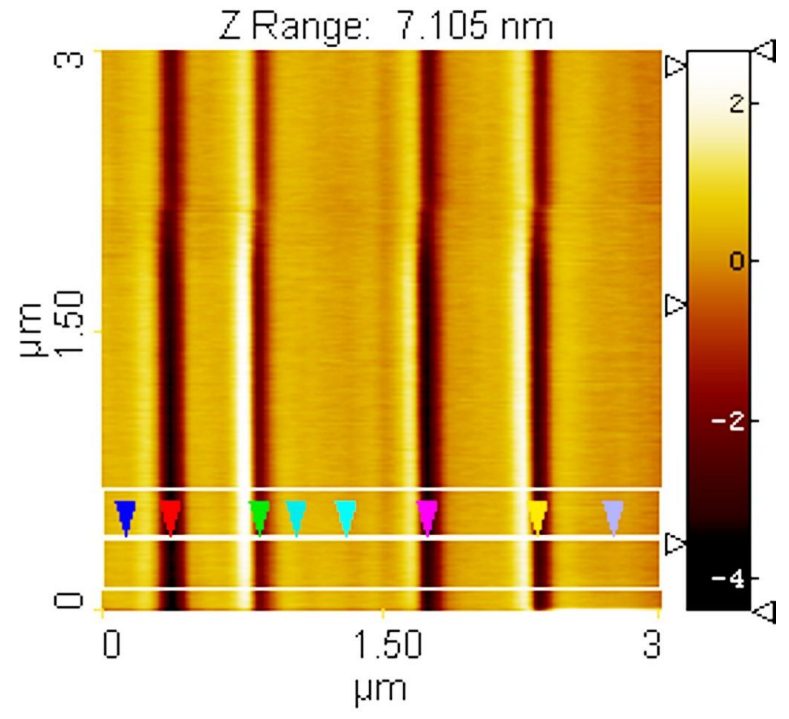

Fig. 2. AFM image of the topography of the area observed on the surface. Markers present the depth of points measured with SPIP program.

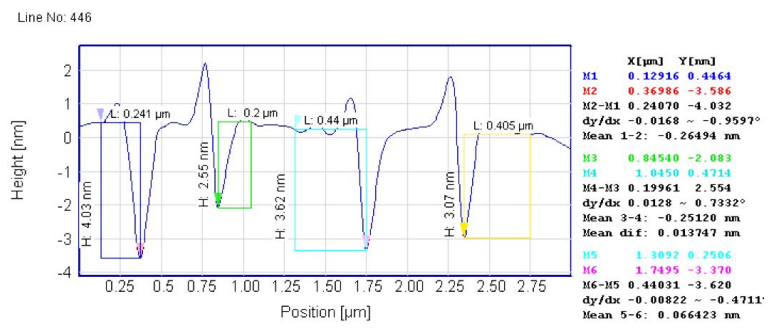

Fig. 3. View of the surface profile and the markers used for depth measurement of each scratch.

the plateau area and the lowest point. In every subsequent measurement of depth, the reference level changes. The method used to obtain the results presented in this paper is a subject of further optimization, and the authors are working to refine it.
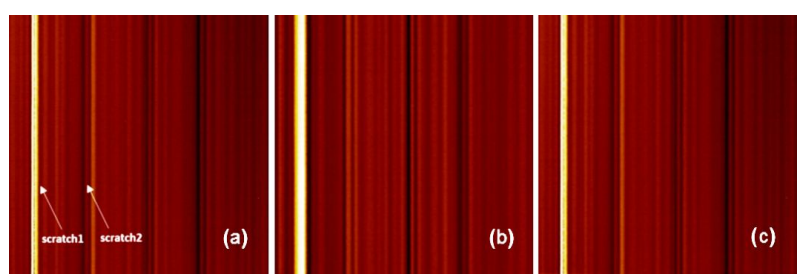

Fig. 4. AFM images of the surface: (a) $1 \mathrm{~min}$, (b) $15 \mathrm{~min}$, (c) $24 \mathrm{~h}$ after nanoscratching. Scan range $3 \mu \mathrm{m} \times 3 \mu \mathrm{m}, \mathrm{Z}$ (height) range: $6.3 \mathrm{~nm}$.

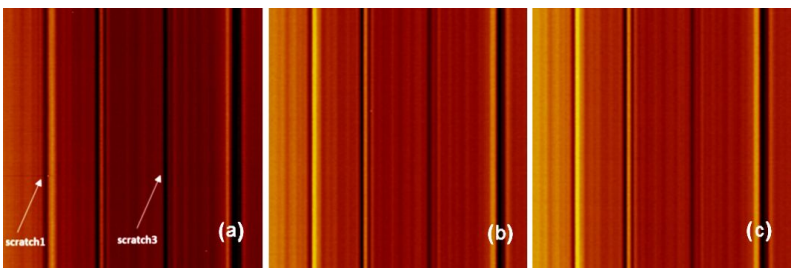

Fig. 5. AFM images of the surface: (a) $1 \mathrm{~min}$, (b) $15 \mathrm{~min}$, (c) $24 \mathrm{~h}$ after nanoscratching. Scan range $3 \mu \mathrm{m} \times 3 \mu \mathrm{m}, \mathrm{Z}$ (height) range: $6.3 \mathrm{~nm}$.

As is shown in Fig. 6, the depth of the scratches decreases over time. However, this phenomenon does not occur in a uniform manner. The graphs do not include the constantly changing width of the resulting cuts, that is visible in Fig. 4 and Fig. 5, the change of which has a substantial impact on the restoration of the passive layer. The lines connecting certain values presented in Fig. 6 have been drawn as an eye-guide to make observation of the dynamics easier. In sample 1, the dynamics of change is very well visible and it appears in a repeatable way, with only slight variations. In contrast, in sample 2, these changes are more diversified, which does not change the fact that, over time, the depth of the cuts decreases. The fact, that the trends shown for sample 1 and sample 2 vary, is caused by the different response of the material at different test points in the sample, resulting from the differentiation in the position of the grains of the examined material [24]. The measurement uncertainty is the result of the evaluation of scatter depth measurement at a given moment, based on statistical analysis. In further research the assessment of all parameters, depth, width and even volume, can be used to assess the dynamics of the processes, which in this scale is possible only by using AFM.

It has been observed that changes in surface modification occur in a continuous manner and the resulting function is differentiable, showing a downward trend and is limited to low noise level. On this basis, we can say that the process of reconstruction of the passivation layer of stainless steel is actually ongoing. It is apparent that this process 


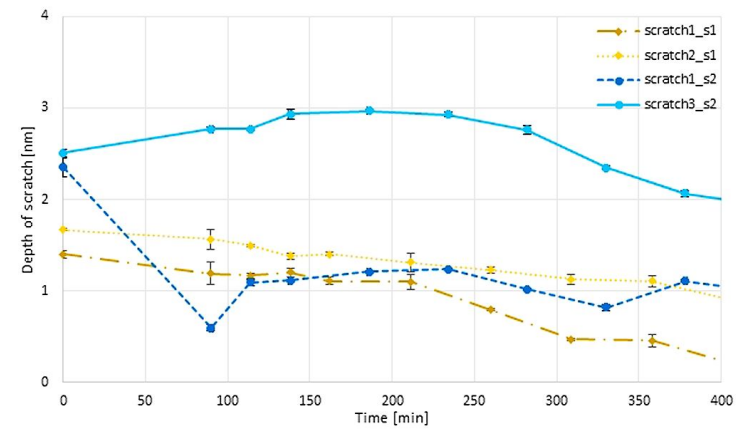

Fig. 6. Relationship between depth of a scratch and time, marked as: (a) sample 1 (scratch1_s1 and scratch2_s1) and (b) sample 2 (scratch1_s2 and scratch3_s2).

is more rapid at the beginning of research and, after some time, that the dynamics of said phenomenon is decreasing.

The above pictures present slight changes that occur in the depth of the tested materials. Samples just after nanoscratching and following the modification after $15 \mathrm{~min}$ and $24 \mathrm{~h}$ were presented. The biggest changes are observed in the first two images of the sample, during first two hours. Visible difference in the behavior of each scratch is also shown. In our study, processes occurring in the same material, but in different grains whose orientation was not the same [24], were observed. Despite the AFM possibilities, finding particles in similar position on the surface is difficult and the development of methodology for such measurements opens a new line of research. Passivation layer properties change at the nano-level depending on the grain, however, they show a similar trend of reducing the depth of the scratches. This is a promising result, which opens the possibility of performing further research in this direction.

\section{Conclusions}

In this paper, we presented the application of advanced AFM technique such as nanoscratching as the method that allows the observation of reconstruction of a passivation layer of stainless steel. Thanks to it we can describe the process of reconstruction in quantitative fashion and to determine its rate at each step of the process. We could observe that its growth rate is greater at the beginning of the study and it diminishes with time. The dynamics of the process also depends on the depth of cut as well as its width. Changing of the feature depth is continuous, decreasing, and is a differentiable function. Using AFM, it is possible to explore these changes, which are not possible to measure on such level with any other measurement technique, due to the requirement of subnanometer detection resolution. It should be emphasized that as the information delivered by this technique provides sub-nanometer resolution of the passivation layer behavior, it is related to local properties of the material. Therefore, as it was stressed out earlier, the grainy structure of the samples may cause the acquisition of the data revealing various trends of the material response. The drawback of the diversity of the curves results in the necessity to obtain and analyze a number of data in order to deliver a statistically reliable information, which is a time consuming process. The advantage of such a situation is the knowledge about the inhomogeneities of the material at a single grains scale, which can be valuable in terms of learning new information about the investigated material. It can also be used for further technology optimization. In order to detect the presence of various grains, force modulation mode, or Kelvin probe force microscopy, can be used as it provides the maps of surface stiffness revealing potential changes on the surface. The most appropriate approach will be tested in order to optimize the measurement technique.

Development of methods facilitating this type of measurements which we will focus on, would allow us to perform further promising research. Size of steel grains also affects material properties. The use of AFM enables observation of how the process of reconstruction occurs, and gives means to develop it in terms of practical applications.

\section{Acknowledgements}

We thank the Electrotechnical Institute for the opportunity to perform research needed for the purposes of this paper. The authors also thank the Association of Polish Electrical Engineers SEP, the Wroclaw Branch, and the Scientific Circle SEP No. 1 in Wroclaw, for their financial support. 


\section{References}

[1] Lochynski P., Kowalski M., Szczygiel B., Kuczews ki K., Pol. J. Chem. Technol., 18 (2016), 76.

[2] Lochynski P., Sikora A., Szczygiel B., Surf. Eng., 33 (5) (2017), 395.

[3] Kosmač A., Elektropolerowanie stali nierdzewnych. Materialy i zastosowania, zeszyt 11, (Electrochemical Treatments of Steel. Materials and Applications, Volume 11), Euro Inox, Brussels, 2009.

[4] Rollet F., Morlat-Thérias S., Gardette L.J., Polym. Degrad. Stabil., 94 (2009), 877.

[5] Nowicki M., Richter A., Wolf B., KaCZMAREK H., Polymer, 44 (2003), 6599.

[6] Sikora A., Grabarek A., Moron L., Walecki M., KRYla P., IOP Conf. Ser., 113 (2016), 12016.

[7] Mikšová R., Macková A., MalinskÝ P., SlepičKa P., Švorčík V., Polym. Degrad. Stabil., 122 (2015), 110.

[8] Suresh B., Maruthamuthu S., Khare A., Palanisamy N., Muralidharan V.S., RaguNATHAN R., Kannan M., PandiYaraj K.N., J. Polym. Res., 18 (2011), 2175.

[9] Ravari F., Omrani A., Rostami A. A., Ehsani M., Polym. Degrad. Stabil., 97 (2012), 929.

[10] Canetta E., Montiel K., Adya A.K., Forensic. Sci. Int., 191 (2009), 6.

[11] RamiaczeK-Krasowska M., SzyszKa A., Prazmowska J., Paszkiewicz R., Tlaczala M., Opt. Appl., 39 (2009), 711.

[12] Bhushan B., Wear, 259 (2005), 1507.
[13] Jang K., Ishibashi Y., Iwata D., Suganuma H. YAMADA T., TAKEMURA Y., J. Nanosci. Nanotechno., 11 (2011), 10945-8.

[14] Ton-That C., Shard A.G., Bradley R.H., Langmuir, 16 (2000), 2281.

[15] SiKora A., Opt. Appl., 43 (2013), 163.

[16] Sikora A., Meas. Sci. Technol., 25 (2014), 055401.

[17] Khurshudov A.G., Kato K., Koide H., Wear, 203 - 204 (1997), 22.

[18] LiU J., Notbohm, J.K., Carpick R.W., Turner K.T., ACS Nano, 4 (2010), 3763.

[19] Su C., HuAng L., KJoller K., BABCock K., Ultramicroscopy, 97 (2003), 135.

[20] Wang H., Xie H., Hu Z., Wu D., Chen P., Polym. Degrad. Stabil., 97 (2012), 1755.

[21] Sikora A., Iwan A., High Perf. Polym., 24 (2012), 218.

[22] https://www.bruker.com/products/ surface-and-dimensional-analysis/ atomic-force-microscopes/modes/modes/ specialized-modes/nanoindent-nanoscratch. html, accessed on: 2017.04.29.

[23] http://www. imagemet.com/products/spip/, accessed on: 2017.03.01.

[24] Zielinski W., Plocinski T., KurzydlowsKi K.J., Mater. Charact., 104 (2015), 42.
Received 2017-03-12 Accepted 2018-06-12 